IOS Press

\title{
Serial treatments of primed low-frequency rTMS in stroke: Characteristics of responders vs. nonresponders
}

James R. Carey*, Huiqiong Deng, Bernadette T. Gillick, Jessica M. Cassidy, David C. Anderson, Lei Zhang and William Thomas

University of Minnesota, Minneapolis, MN, USA

\begin{abstract}
.
Purpose: This study analyzed the characteristics of responders vs. nonresponders in people with stroke receiving a novel form of repetitive transcranial magnetic stimulation (rTMS) to improve hand function.

Methods: Twelve people with stroke received five treatments of 6-Hz primed low-frequency rTMS to the contralesional primary motor area. We compared demographic factors, clinical features, and the ipsilesional/contralesional volume ratio of selected brain regions in those who improved hand performance $(\mathrm{N}=7)$ on the single-hand component of the Test Évaluant la performance des Membres supérieurs des Personnes Âgées (TEMPA) and those who showed no improvement ( $\mathrm{N}=5$ ).

Results: Responders showed significantly greater baseline paretic hand function on the TEMPA, greater preservation volume of the ipsilesional posterior limb of the internal capsule (PLIC), and lower scores (i.e. less depression) on the Beck Depression Inventory than nonresponders. There were no differences in age, sex, stroke duration, paretic side, stroke hemisphere, baseline resting motor threshold for ipsilesional primary motor area (M1), NIH Stroke Scale, Upper Extremity Fugl-Meyer, Mini-Mental State Examination, or preservation volume of M1, primary somatosensory area, premotor cortex, or supplementary motor area. Conclusion: Our results support that preserved PLIC volume is an important influential factor affecting responsiveness to rTMS.
\end{abstract}

Keywords: stroke, repetitive transcranial magnetic stimulation, posterior limb internal capsule, primary motor area

\section{Introduction}

The loss of hand control after stroke is due not only to the damage caused by the vascular insult but also to downregulation of surviving neurons. This downregulation stems in part from maladaptive changes in interhemispheric inhibition (IHI) in people with stroke. Within limits, IHI is the normal physiological feature by which neurons from the primary motor cortex (M1) initiating a motor command to the intended hand also send signals through the corpus callosum that inhibit neurons in the contralateral M1 (Ferbert et al. 1992),

\footnotetext{
*Corresponding author: James R. Carey, PhD, PT, FAPTA, Program in Physical Therapy, University of Minnesota, MMC Box 388, 420 Delaware St. SE, Minneapolis, MN 55455, USA. Tel.: +612 626 2746; Fax: +612 625 4274; E-mail: carey007@umn.edu.
}

putatively to prevent mirroring movements in the unintended hand (Hinder et al. 2010). However, IHI can become exaggerated in stroke in the direction of contralesional M1 suppressing surviving motor neurons in ipsilesional M1 (Murase et al. 2004; Grefkes et al. 2010; Kirton et al. 2010), thereby magnifying the functional deficit (Khedr et al. 2009; Khedr and Fetoh 2010). To correct this maladaptation, studies have used suppressive low-frequency repetitive transcranial magnetic stimulation (rTMS) over contralesional M1 to disinhibit ipsilesional M1 and promote higher function in the paretic hand (Mansur et al. 2005; Takeuchi et al. 2005; Fregni et al. 2006; Liepert et al. 2007; Dafotakis et al. 2008; Nowak et al. 2008; Takeuchi et al. 2008; Khedr et al. 2009; Emara et al. 2010; Grefkes et al. 2010). 
Importantly, not all brains behave the same and there can be considerable inter-individual variability in the physiologic response to rTMS, as demonstrated in healthy subjects (Maeda et al. 2000b). Indeed, there are occasional reports in patients with stroke of no responsiveness to $1-\mathrm{Hz}$ rTMS applied contralesionally (Theilig et al. 2011; Seniów J 2012) and to continuous theta burst stimulation (another suppressive neuromodulation approach) applied contralesionally (Talelli et al. 2012). Furthermore, studies have shown that suppressive neuromodulation to contralesional M1 in certain patients with stroke can actually decrease function in the paretic upper limb (Lotze et al. 2006; Ackerley et al. 2010; Bradnam et al. 2012). Relatedly, TMS to the contralesional dorsal premotor cortex (PMd) at rest has been shown to be suppressive to ipsilesional M1 excitability in higher functioning patients but it was less suppressive or even facilitatory in lower functioning patients (Bestmann et al. 2010). These combined results suggest that contralesional M1 and PMd may serve an important adaptive role in preserving a modicum of ipsilesional corticospinal function in patients with the greatest impairment. But the stroke characteristics that lead to these and other adaptive changes in some and different changes in others remain for the most part unknown. Such variable reactions to neuromodulation across patients with stroke emphasizes that there is a critical need for research that explores the specific factors in stroke that influence responsiveness to neuromodulation intervention.

Accordingly, the purpose of this descriptive study was to compare the characteristics of responders vs. nonresponders in people with stroke receiving $6-\mathrm{Hz}$ primed low-frequency rTMS. We included 6-Hz priming to accentuate the effects of the low-frequency rTMS, as shown in healthy subjects (Iyer et al. 2003), which is based on principles of homeostatic plasticity (Siebner et al. 2004; Ilic et al. 2011; Cassidy et al. 2014). Exploring the characteristics of responders vs. nonresponders will set the stage for a future research comparing primed vs. unprimed low-frequency rTMS in stroke.

\section{Materials and methods}

\subsection{Subjects}

Twelve people with stroke were enrolled in the study and all received active rTMS. These subjects

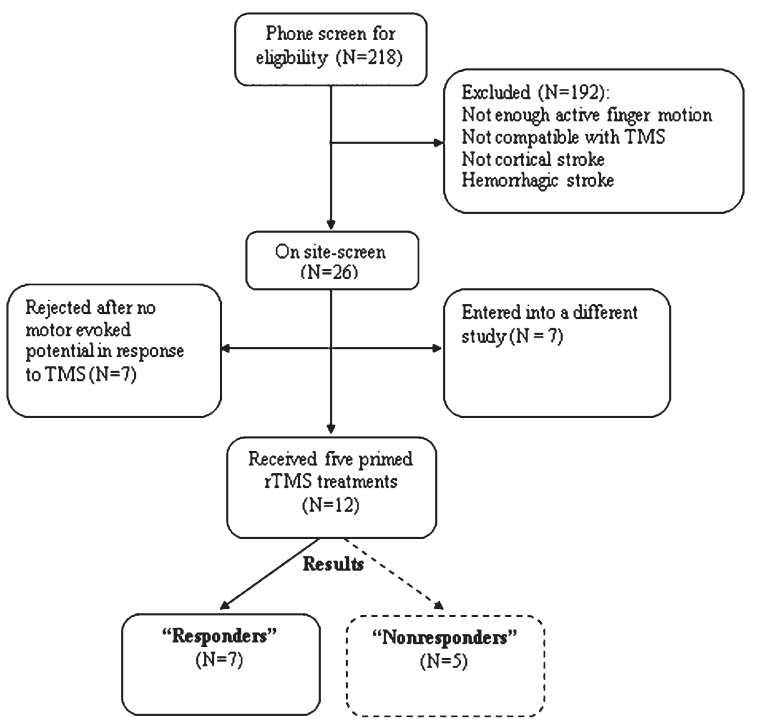

Fig. 1. Enrollment of subjects and formation of responder vs. nonresponder groups to repetitive transcranial magnetic stimulation (rTMS).

were ultimately divided into two groups, responders or nonresponders, based on change in behavioral performance following rTMS (see 2.7 Analysis and Fig. 1). Inclusion criteria were ischemic cortical stroke $>6$ months duration, $>10$ degrees of active finger extension, and elicitation of a motor evoked potential (MEP) with TMS over ipsilesional M1. The rationale for requiring active finger extension and an ipsilesional MEP was to study a more homogeneous sample of subjects with characteristics that offered a seemingly higher chance for improving paretic hand function. Previous work has shown that the presence of an elicitable MEP is more favorable to higher recovery of function (Talelli et al. 2006; Dimyan and Cohen 2010). Exclusion criteria were history of seizures and indwelling metal or devices incompatible with rTMS. No subjects were receiving formal therapy. None of the subjects were on centrally-acting medications during their involvement in this study. Individual characteristics are shown in Table 1 and comparisons are reported in Results. This study was approved by the Institution's Committee on the Use of Human Subjects in Research and all subjects gave informed consent.

\subsection{Magnetic Resonance Imaging}

To confirm stroke location and rule out hemorrhage, fluid attenuated inversion recovery (FLAIR) and 
Table 1

Subject characteristics

\begin{tabular}{|c|c|c|c|c|c|c|c|c|c|c|}
\hline Subject & Sex & $\begin{array}{c}\text { Age } \\
\text { (years) }\end{array}$ & $\begin{array}{l}\text { Stroke } \\
\text { Location }\end{array}$ & $\begin{array}{l}\text { Stroke Hemisphere } \\
\text { (relative to preferred } \\
\text { Hand before stroke) }\end{array}$ & $\begin{array}{c}\text { Stroke } \\
\text { Duration } \\
\text { (months) }\end{array}$ & UEFM & MMSE & BDI & NIHSS & $\begin{array}{c}\text { Responder } \\
\text { Vs. } \\
\text { Nonresponder }\end{array}$ \\
\hline 1 & $\mathrm{M}$ & 70 & $\begin{array}{l}\text { R temporal, frontal, } \\
\text { parietal lobes, insula, } \\
\text { internal capsule }\end{array}$ & Ipsilateral & 118 & 35 & 27 & 14 & 2 & Nonresponder \\
\hline 2 & $\mathrm{M}$ & 59 & $\begin{array}{l}\text { L parietal lobe, insula, internal } \\
\text { capsule }\end{array}$ & Contralateral & 58 & 54 & 23 & 1 & 1 & Responder \\
\hline 3 & $\mathrm{~F}$ & 53 & $\begin{array}{l}\mathrm{R} \text { frontal, parietal, temporal } \\
\text { lobes, internal capsule }\end{array}$ & Ipsilateral & 47 & 29 & 30 & 4 & 4 & Responder \\
\hline 4 & $\mathrm{~F}$ & 82 & $\begin{array}{l}\text { L frontal, parietal lobes, internal } \\
\text { capsule }\end{array}$ & Ipsilateral & 75 & 53 & 30 & 7 & 5 & Responder \\
\hline 5 & M & 54 & $\begin{array}{l}\mathrm{R} \text { temporal, frontal lobes, internal } \\
\text { capsule }\end{array}$ & Ipsilateral & 31 & 42 & 28 & 13 & 4 & Nonresponder \\
\hline 6 & M & 79 & L frontal lobe, internal capsule & Contralateral & 48 & 31 & 26 & 8 & 3 & Responder \\
\hline 7 & M & 51 & $\begin{array}{l}\text { L frontal, parietal lobes, internal } \\
\text { capsule }\end{array}$ & Contralateral & 34 & 23 & MD & 8 & 7 & Nonresponder \\
\hline 8 & $\mathrm{~F}$ & 65 & $\begin{array}{l}\mathrm{R} \text { frontal, parietal, temporal } \\
\text { lobes, internal capsule }\end{array}$ & Ipsilateral & 33 & 31 & MD & 12 & 8 & Nonresponder \\
\hline 9 & $\mathrm{M}$ & 58 & $\mathrm{R}$ frontal lobe, internal capsule & Ipsilateral & 44 & 57 & 30 & 10 & 4 & Responder \\
\hline 10 & $\mathrm{M}$ & 73 & $\mathrm{~L}$ parietal lobe & Contralateral & 78 & 58 & 24 & 5 & 4 & Responder \\
\hline 11 & $\mathrm{~F}$ & 54 & $\mathrm{R}$ frontal, temporal lobes & Ipsilateral & 29 & 46 & 30 & 22 & 4 & Nonresponder \\
\hline 12 & M & 55 & L frontal lobe, internal capsule & Ipsilateral & 15 & 22 & 19 & 4 & 6 & Responder \\
\hline
\end{tabular}

rTMS = Repetitive transcranial magnetic stimulation, $M=$ male, $F=$ female, $R=$ right, $L=$ Left, UEFM=Upper Extremity Fugl-Meyer $(0-66$, best $=66)$, MMSE $=$ Mini-Mental State Examination $(0-30$, best $=30)$, BDI $=$ Beck Depression Inventory $(0-63$, best $=0)$, NIHSS $=$ National Institutes of Health Stroke Scale $(0-42$, best $=0), M D=$ missing data.

gradient echo magnetic resonance imaging was performed on each subject inside a 3-Tesla magnet (Magnetom Trio, Siemens, Munich, Germany) equipped with a standard 12-channel head coil. These images were reviewed by a neurologist. In addition, highresolution $\left(1-\mathrm{mm}^{3}\right)$, T1-weighted, anatomical images (3D FLASH, TR 20 milliseconds, FA $30^{\circ}$, acquisition time $=5: 00$ minutes) were acquired to allow volumetric analysis (voxel count) of M1, primary somatosensory area (S1), premotor cortex (PMC), supplementary motor area (SMA) and the posterior limb of the internal capsule (PLIC) for each hemisphere using Brain Voyager QX (Brain Innovation B.V., Maastricht, Netherlands) software. For M1, S1, PMC and SMA we used landmarks specified by Dassonville et al. (2001). For PLIC, the landmarks were the most superior aspect of the lenticular nucleus superiorly, anterior commissure inferiorly, genu of the internal capsule anteriorly, posterior edge of the putamen posteriorly, lenticular nucleus laterally, and thalamus medially (Schaechter et al. 2008). For each region of interest, the ratio of ipsilesional/contralesional (I/C) volume was computed. We did attempt to use diffusion tensor imaging as a more sophisticated morphometry approach, however, head movement greater than $1 \mathrm{~mm}$ occurred during acquisi- tion in essentially all subjects that was of a magnitude $(>1 \mathrm{~mm})$ that precluded effective DTI analysis but was tolerable $(3-5 \mathrm{~mm})$ for conventional analysis.

\subsection{Screening for stroke characteristics}

We administered certain tests only at baseline to explore descriptive characteristics that might influence responders vs. nonresponders to treatment. These tests included the National Institutes of Health Stroke Scale (NIHSS) (Bates et al. 2005) as an indicator of stroke severity and lateralized function, the upper-extremity Fugl-Meyer (UEFM) test (Fugl-Meyer et al. 1975) as an indicator of upper limb impairment, the Edinburgh inventory for preferred hand prior to stroke (Oldfield 1971), the Minimental State Exam (MMSE) (Folstein et al. 1975) as an indicator of cognition, the Beck Depression Inventory II (BDI)(Beck et al. 1961; Berg et al. 2003) as an indicator of depression, and the resting motor threshold (RMT) for ipsilesional M1 as an indicator of corticospinal excitability.

The RMT for ipsilesional M1 was found using a Magstim 200 magnetic stimulator (Magstim Company Limited, Dyfed, UK) and a 70-mm figure-of-eight coil. We monitored responses from the paretic exten- 
sor digitorum (ED) muscle using adhesive electrodes. The active electrodes were positioned in parallel with the muscle in a bipolar arrangement one $\mathrm{cm}$ apart at the midpoint between the lateral epicondyle of the humerus and the dorsal tubercle of the radius. The reference electrode was secured to the dorsum of the hand. The electrodes were connected to a Cadwell Sierra Wedge EMG amplifier (Cadwell Laboratories, Kennewick, WA). The bandpass was $20 \mathrm{~Hz}-2 \mathrm{kHz}$. We used a $60-\mathrm{Hz}$ notch filter. The sampling rate was $6.4 \mathrm{kHz}$. The gain was set to $50 \mu \mathrm{V} /$ division. The sweep was set to $10 \mathrm{~ms} /$ division. EMG signals were collected for $10 \mathrm{~ms}$ prior to and $90 \mathrm{~ms}$ following the TMS pulse. As our intent was to identify the RMT, any trials showing EMG activity prior to the stimulus artifact were discounted. Subjects rested in a recliner chair in a semi-recumbent position. Subjects wore earplugs and a tight-fitting Lycra swim cap (Invista, Inc., Wilmington, Delaware) to allow markings. The coil handle was directed posterolaterally $45^{\circ}$ to the mid-sagittal line. The coil location and stimulus intensity were adjusted until the hotspot and RMT (lowest intensity producing an MEP of at least $50 \mu \mathrm{V}$ peak-to-peak on at least 5 of 10 trials) was found (Butefisch et al. 2004).

\subsection{Intervention}

Prior to rTMS intervention, the hotspot and RMT for contralesional M1 were found using the same procedures as described above except that the target muscle was the nonparetic ED. The RMT for contralesional M1 was determined anew each treatment day. Priming rTMS was applied using a Magstim Rapid ${ }^{2}$ stimulator and a 70-mm Air Film Coil. Priming consisted of 10 minutes of intermittent $6-\mathrm{Hz}$ rTMS given in 5-s trains at 2 trains per minute with 25-s intervals between trains (total pulses $=600$ ) at an intensity of $90 \%$ RMT. Then, 10 minutes of continuous $1-\mathrm{Hz}$ rTMS (total pulses $=600$ ) was given at $90 \%$ RMT. This procedure was repeated every other weekday for five sessions.

To evaluate training vs. no training as a factor affecting the responsiveness to rTMS, subjects were randomly assigned to receive either rTMS+tracking training or rTMS+no training on the days that alternated with the rTMS. For each tracking session, we attached a custom electrogoniometer to the paretic index finger. Subjects viewed a computer screen displaying target waveforms and performed 120 extension/flexion tracking trials over 30 minutes, as described earlier (Carey et al. 2002).

\subsection{Outcome Measure}

Our primary outcome measure was the performance time on the single-hand component of the Test Évaluant la performance des Membres supérieurs des Personnes Âgées (TEMPA) (Desrosiers et al. 1995), which is a reliable measure of manual skill (Moseley and Yap 2003). The single-hand component consists of four tasks: 1) grasp and move a jar, 2) pour water from a pitcher into a glass and move glass to mouth, 3) pick up coins and deposit into a slot, and 4) grasp and release small objects. Completion times (maximum allowed time for each task was 120 seconds) were recorded for these tasks with the paretic hand alone TEMPA $\left._{\text {paretic }}\right)$ and the nonparetic hand alone (TEMPA $\left.{ }_{\text {nonparetic }}\right)$. The TEMPA $_{\text {nonparetic }}$ was used as a safety measure, exploring for diminished skill in the nonparetic hand. Testing occurred at baseline and the first weekday following the last treatment (posttest).

\subsection{Safety}

As this was the first study to apply multiple sessions of 6-Hz priming of low-frequency rTMS, we also analyzed safety. All subjects were combined for safety analysis. A neurologist conducted a clinical examination of neurological function in all subjects at baseline, each treatment day, and posttest. Motor performance in the nonparetic hand was assessed with

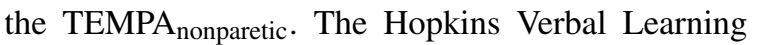
Test-Revised (HVLT-R) (Brandt and Benedict 2001), which required subjects to remember words on a spoken list, measured possible adverse cognitive effects from one treatment day to the next. Lastly, at each treatment session and at posttest, subjects were questioned on whether they experienced any abnormal symptoms that may have occurred after the previous session. The specific questions addressed seizure, headache, neck pain, dental pain, hearing deficit, nausea, muscle contractions, sleep disturbance, altered concentration, anxiety, memory difficulty, disturbed mood, balance problems, impairment of the strong hand, or any other problem (Carey et al. 2008).

\subsection{Analysis}

After intervention, we divided the 12 subjects, all of whom received rTMS, into two groups, responders vs. nonresponders. We defined responders as those showing any improvement (reduction in total time) 
on the primary dependent measure, the TEMPA paretic $_{\text {. }}$ Nonresponders were defined as those showing no improvement. We then used two-sample $t$ tests to determine whether there was a significant difference between responders and nonresponders at baseline and whether the change at posttest was significantly different between them. Next, we extended the responder vs. nonresponder analysis by comparing demographic, stroke, and training factors between groups using twosample $t$ tests for interval/ratio data and Fisher's exact test for categorical data. For data not meeting normality and equal-variance assumptions and for ordinal data, Mann Whitney U tests were used. Statistical significance was determined at $p=0.05$. We did not correct for multiple comparisons on the principle that the restrictiveness of Bonferroni correction could hinder the advancement of the field in initial exploratory studies with low N (Pocock 1997; Nakagawa 2004). All data were analyzed using NCSS/2007 (NCSS, Kaysville, Utah).

\section{Results}

\subsection{Responders vs. nonresponders}

Of 12 subjects, 7 improved their TEMPA paretic $_{\text {score }}$ from baseline to posttest and were classed as "responders;" the remaining 5 did not improve and were classed as "nonresponders." TEMPA each subject at baseline and posttest are shown in Fig. 2A. In the paretic hand of responders, mean (SD) time to completion of the TEMPA paretic $_{\text {at }}$ baseline was 185.5 (138.8) seconds (s), which was significantly less than the 458.4 (48.3) s for nonresponders $(p=0.002)$. Responders improved in performance by 69.8 (75.5) $\mathrm{s}$ from baseline to posttest, whereas nonresponders declined in performance by $4.5(10.1) \mathrm{s}(\mathrm{p}=0.005)$.

In the nonparetic hand of responders, mean TEMPA $_{\text {nonparetic }}$ performance at baseline was 42.2 (6.1) $\mathrm{s}$, which was not significantly different from the $37.9(9.1)$ s performance of nonresponders $(\mathrm{p}=0.344)$. Responders improved slightly by $1.5(8.5) \mathrm{s}$ at posttest as did the nonresponders by $3.5(5.5) \mathrm{s}$ with the difference in change between groups being nonsignificant $(p=0.643)$. TEMPA nonparetic scores for each subject at baseline and posttest are shown in Fig. 2B.

We further explored performance by normalizing the TEMPA paretic $_{\text {score to the TEMPA }}$ nonparetic score and comparing this ratio from baseline to posttest. The

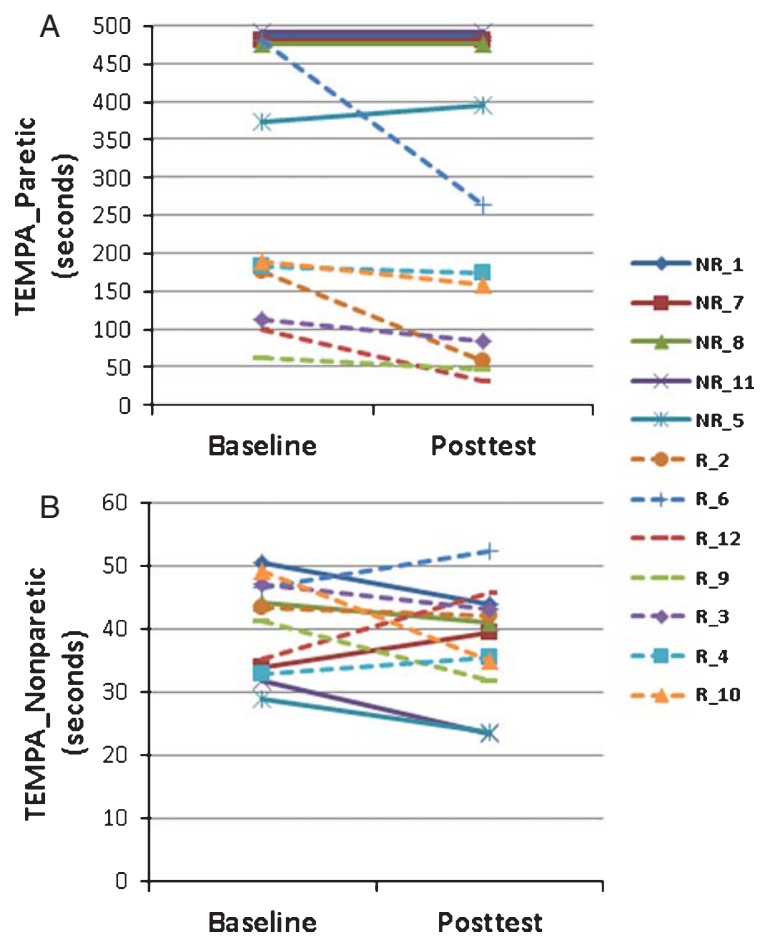

Fig. 2. Baseline and posttest times for each responder ( $\mathrm{R}$, dashed lines) and nonresponder (NR, solid lines) on the Test Évaluant la performance des Membres supérieurs des Personnes Âgées (TEMPA) for the four tasks isolated to the paretic hand (A) and nonparetic hand (B). The upper limit of time was cut off at 120 seconds for each task, which occurred for four nonresponders, hence their lines are superimposed.

mean pretest $\mathrm{TEMPA}_{\text {paretic }} / \mathrm{TEMPA}_{\text {nonparetic }}$ ratio was 4.3 (2.9) for responders vs. 12.5 (2.3) for nonresponders $(\mathrm{p}<0.001)$. For responders at posttest, the mean ratio decreased by $1.5(2.0)$, whereas for nonresponders the ratio increased by 1.9 (2.9) with the difference between responders and nonresponders being significant $(\mathrm{p}<0.035)$. Thus, following intervention, mean performance in the paretic hand of responders moved closer to their nonparetic hand, whereas for nonresponders it did not.

The comparison between responders and nonresponders in Table 2 showed no significant differences in the factors of age, sex, stroke duration, paretic side, stroke hemisphere (i.e. contralateral or ipsilateral to preferred hand before stroke), baseline ipsilesional M1 RMT, treatment (rTMS+tracking training or rTMS+no training), NIHSS, UEFM, MMSE. Also, there were no significant differences in the I/C ratios for M1, S1, PMC, or SMA. To the contrary, responders did show 
Table 2

Comparison [mean (SD)] of responder vs. nonresponder characteristics

\begin{tabular}{|c|c|c|c|c|}
\hline Baseline characteristic & Statistical test & Responder $(N=7)$ & Nonresponder $(N=5)$ & $P$ value \\
\hline Age (years) & Two-sample t & $65.6(12.2)$ & $58.8(8.29)$ & 0.31 \\
\hline Sex ( $\mathrm{N}$ females/ $\mathrm{N}$ males) & Fisher & $2 / 5$ & $2 / 3$ & 0.99 \\
\hline Stroke duration (months) & Two-sample t & $52.1(21.2)$ & $49.0(38.8)$ & 0.86 \\
\hline Paretic side ( $\mathrm{N}$ left/N right) & Fisher & $2 / 5$ & $4 / 1$ & 0.24 \\
\hline $\begin{array}{l}\text { Stroke hemisphere ( } \mathrm{N} \text { contralateral/ } \mathrm{N} \\
\text { ipsilateral to preferred hand before stroke) }\end{array}$ & Fisher & $3 / 4$ & $3 / 4$ & 0.58 \\
\hline $\begin{array}{l}\text { Ipsilesional M1 RMT (\% of machine } \\
\text { maximum) }\end{array}$ & Two-sample t & $51.6(23.0)$ & $52.0(9.7)$ & 0.97 \\
\hline $\begin{array}{l}\text { Treatment }(\mathrm{N} \text { receiving } \mathrm{rTMS}+\text { tracking } \\
\text { training/ } \mathrm{N} \text { receiving } \mathrm{rTMS}+\text { no training })\end{array}$ & Fisher & $3 / 4$ & $3 / 2$ & 0.99 \\
\hline NIHSS [median (interquartile range)] & Mann Whitney U & $4.0(2.0)$ & $4.0(4.5)$ & 0.5 \\
\hline UEFM & Two-sample t & $43.4(15.4)$ & $35.4(9.2)$ & 0.32 \\
\hline MMSE & Two-sample t & $26.0(4.24)$ & $28.3(2.0)$ & 0.4 \\
\hline BDI & Two-sample t & $5.6(2.9)$ & $13.8(5.2)$ & 0.005 \\
\hline M1 volume I/C ratio & Two-sample t & $0.79(0.27)$ & $0.86(0.16)$ & 0.62 \\
\hline $\mathrm{S} 1$ volume $\mathrm{I} / \mathrm{C}$ ratio & Two-sample t & $0.92(0.42)$ & $0.82(0.21)$ & 0.64 \\
\hline PMC volume I/C ratio & Two-sample t & $1.11(0.36)$ & $0.95(0.15)$ & 0.37 \\
\hline SMA volume I/C ratio & Two-sample t & $0.84(0.28)$ & $0.97(0.32)$ & 0.47 \\
\hline PLIC volume I/C ratio & Two-sample t & $0.85(0.16)$ & $0.25(0.16)$ & $<0.001$ \\
\hline
\end{tabular}

RMT $=$ resting motor threshold, NIHSS = National Institutes of Health Stroke Scale, UEFM = Upper Extremity Fugl-Meyer, MMSE = Minimental State Exam Score, BDI = Beck Depression Inventory II, M1 = primary motor area, S1 = primary somatosensory area, PMC = premotor cortex, $\mathrm{SMA}=$ supplementary motor area, PLIC = posterior limb internal capsule, $\mathrm{I} / \mathrm{C}=$ Ipsilesional/Contralesional.

significantly lower scores (i.e. less depression) on the $\mathrm{BDI}(\mathrm{p}=0.005)$ and a significantly greater $\mathrm{I} / \mathrm{C}$ ratio for the PLIC $(\mathrm{p}<0.001)$.

Fig. 3 gives a visual representation of the amount of sparing of ipsilesional M1 and PLIC across all subjects in each group and points to the greater influence of PLIC compared to M1.

\subsection{Safety}

No decline in neurological examination was

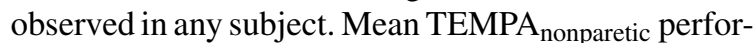
mance did not decline (See Section 3.1 and Fig. 2B). Immediate recall of words on the HVLT-R was 21.2 (5.9) at baseline and $21.1(6.7)$ at posttest $(\mathrm{p}=0.907)$. Delayed recall was 6.8 (2.6) words at baseline and 5.4 (3.6) at posttest, which was a significant difference $(\mathrm{p}=0.049)$. Retention \% was 81.9 (24.9) at baseline and $61.3(33.8)$ at posttest $(\mathrm{p}=0.097)$. Discrimination was 9.0 (9.7) words at baseline and 9.0 (9.8) at posttest $(\mathrm{p}=0.880)$. Inspection of the delayed recall data revealed that the decline in mean values were influenced mainly by one subject, \#6, who had a delayed recall of only one word at posttest compared to six words at baseline. But this person reported no memory or concentration difficulties upon questioning at posttest nor did the neurologist identify any gross mental changes. A follow-up conversation with this person indicated no memory or concentration difficulties. Accordingly, this response was graded as a mild adverse event (Trotti et al. 2003).

At daily interview, the symptoms and number of subjects reporting them at one or more treatment sessions included fatigue (3), itching on foot (1), neck stiffness (1), shoulder pain (1), difficulty concentrating (1), tired paretic hand (1), muscle twitching (1), and headache (1). All symptoms resolved spontaneously within 24 hours of onset.

\section{Discussion}

This study explored possible factors influencing the behavioral responsiveness to $6-\mathrm{Hz}$ primed lowfrequency rTMS to contralesional M1. The important factors that emerged were baseline paretic hand function, the amount of preservation of the ipsilesional PLIC, and depression score on the BDI.

Our finding that the amount of preservation of the PLIC is related to responsiveness to rTMS is consistent with other studies showing that greater integrity of the corticospinal tract is important to stroke recovery (Lindenberg et al. 2010; Zhu et al. 2010; Nouri and Cramer 2011). Importantly, however, our results did not show the same value for the integrity of 


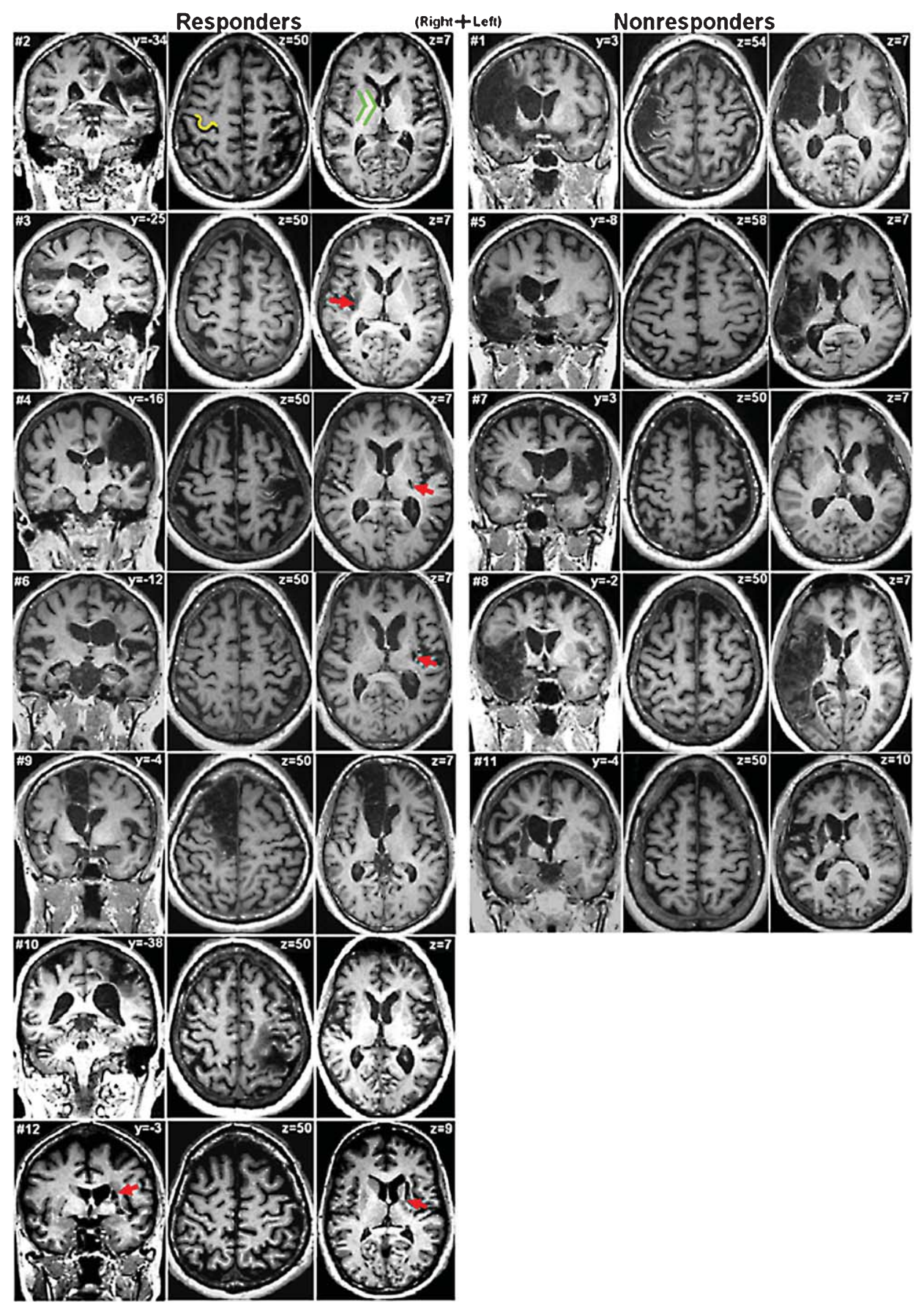

Fig. 3. Magnetic resonance images for responders contrasted to nonresponders. For each subject, the left image shows the coronal slice at the level where the infarct is largest. The middle image shows a transverse slice at the level of the "hand knob" (Yousry et al. 1997) of primary motor area (M1), exemplified in subject \#2 as yellow. The right image shows a lower transverse slice at the level of the internal capsule, exemplified in green. Not all transverse images show infarcts. Red arrows mark difficult-to-see infarcts. Responder status appears to be better indicated by posterior limb of internal capsule (PLIC) involvement than by M1 involvement. Slice $z=50$ of subject \#4 shows an infarct that largely affects M1, including some of the hand knob, yet this subject was a responder, likely because of only small involvement of the PLIC (slice $z=7$ ). Conversely, nearly all the nonresponders (except subject \#1) show no involvement of M1 at the level of the hand knob but substantial involvement of the PLIC (including subject \#1). 
the ipsilesional motor cortices (M1, PMC, SMA), which is also consistent with the assessments of others (Ward et al. 2006; Hamzei et al. 2008; Riley et al. 2011). Figure 3 shows a wide range of stroke lesions, some largely affecting ipsilesional M1 of responders and, equally important, some showing small lesions in ipsilesional M1 of nonresponders. The functional deficit in the paretic hand likely stemmed from both the death of infarcted cortical motor neurons and the downregulated excitability of more distant, surviving motor neurons in M1, PMC, and SMA (i.e. diaschisis) (Feeney and Baron 1986). For the responders then, the improved behavior following rTMS likely stemmed from upregulating spared cortical neurons in M1 and possibly the adjacent PMC and SMA where hand function can be promoted vicariously (Rossini and Pauri 2000; Jaillard et al. 2005; Dancause 2006; Schulz et al. 2012). But such upregulated activity can only manifest into improved behavioral function if the descending projections remain intact. For the nonresponders, any rTMS-induced upregulation was ineffectual, likely due to their large loss of PLIC integrity, thereby preventing descending projections from surviving M1 primarily and PMC and SMA secondarily connecting with spinal motor neurons (Schulz et al. 2012), and less likely to loss of cortex integrity. This speculation needs to be confirmed in future experiments that quantify cortical lesion volumes and interhemispheric inhibition. Noteworthy, a meta-analysis showing that patients with subcortical stroke responded more favorably to rTMS than patients with cortical stroke (Hsu et al. 2012) does not contradict the above findings emphasizing the importance of PLIC. Many subcortical strokes do not involve PLIC.

Thus, integrity of the PLIC appears to be a crucial stroke characteristic in identifying which patients with motor deficits are most apt to respond to rTMS and other neuromodulation interventions. More research is now needed to quantify the critical mass of preserved PLIC to optimize identification of appropriate candidates for rTMS. Even if the problems with head movement in people with stroke could be prevented during DTI, because of the sophistication required in DTI analysis, it seems unlikely, at least currently, that DTI will be available in many medical centers to assess PLIC integrity and patient candidacy for neuromodulation. More practically, clinicians should begin to screen and quantify the fraction of preserved PLIC relative to the unaffected side using standard anatomical images and imaging software to deduce the critical mass needed to decide who should receive and not receive neuromodulation, similar to indices being developed with DTI (Stinear et al. 2007; Stinear et al. 2012). Such unequal treatment across patients broaches a difficult ethical topic but it must be addressed with the realization that "one size does not fit all"(Bradnam et al. 2012) and to deny treatment to appropriate candidates to ensure equality for all has its own ethical dilemmas.

We speculate that variability in responsiveness with neuromodulation is probably greater with suppressive neuromodulation to contralesional M1 than with facilitatory neuromodulation to ipsilesional M1. Whereas suppression of IHI can disinhibit ipsilesional M1 and improve function in some people with stroke, other people with stroke with greater impairments appear to adapt differently and depend on interhemispheric connections to up-regulate secondary motor pathways, such that suppression of their interhemispheric connections can result in deteriorated function rather than enhanced function (Lotze et al. 2006; Ackerley et al. 2010; Bradnam et al. 2012). Nonetheless, suppressive neuromodulation to contralesional M1 should still continue, in appropriate patients, as the aforementioned meta-analysis has shown that suppressive rTMS to contralesional M1 was more efficacious than facilitatory rTMS to ipsilesional M1(Hsu et al. 2012). We surmise that candidate appropriateness for contralesional neuromodulation hinges heavily on the amount of PLIC preservation.

Our finding that subjects with lower (better) baseline

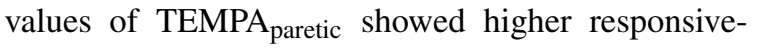
ness to rTMS is consistent with the observation that the same subjects also showed greater PLIC preservation, reflecting the functional importance of PLIC preservation. Conversely, baseline performance on the Fugl-Meyer was not a characteristic differentiating responders from nonresponders. We submit that the upper extremity Fugl-Meyer evaluates impairment,

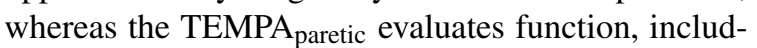
ing finger fractionation. As the rTMS was targeted to improve finger function, it is not surprising that the Fugl-Meyer did not differentiate between responders and nonresponders. As with PLIC preservation, further research is needed to identify the most sensitive finger task and the critical level of baseline performance to accurately and ethically guide decision making for rTMS candidates.

The finding that higher BDI scores (more depressed mood) was a deterrent to rTMS responsiveness is consistent with other studies showing that depression 
has negative effects on functional recovery in stroke (Chemerinski et al. 2001; Gainotti et al. 2001; Paolucci et al. 2001; Pohjasvaara et al. 2001; Hackett and Anderson 2005). The possibility exists that patients with higher levels of depression may have reduced motivation to perform tasks and also that they may be less susceptible to the benefits of placebo effects. Although it is intuitive that a depressed state of mind may be associated with diminished recovery of physical function, the cause vs. effect conundrum, for the most part, has not been made clear (Robinson and Spalletta 2010). However, Parikh et al. (1990) compared depressed vs. non-depressed patients in the very early stage following stroke when functional deficits were similar. They found that despite similar amounts of therapy between groups, the depressed group showed less recovery at two-year follow-up, suggesting that depression has a real effect on functional outcome rather than functional outcome having an effect on depression. Furthermore, Narushima and Robinson (2003) evaluated early vs. delayed antidepressant treatment in people with stroke. They showed that, despite similar amounts of rehabilitation, those who received early antidepressant treatment had greater functional recovery at 12- and 24-month follow-up tests than those receiving the same antidepressant treatment but delayed in time. The relevance to rTMS is that there may be genetic factors that predispose some individuals to post-stroke depression (Kim et al. 2012a) and time-dependent treatment (Narushima and Robinson 2003), which may also influence neuroplasticity and responsiveness to rTMS. Although speculative, this invites further research.

Our work suggests that age, sex, stroke hemisphere, duration of stroke, NIHSS, cognition and tracking training were not influential factors in affecting responsiveness to rTMS for the subjects of this study. More research is needed, however, as our sample size was small yielding low statistical power for uncovering other influential stroke characteristics that might truly exist. Moreover, as an inclusion criterion, all of our subjects had stroke for $>6$ months; the results may be different for acute or subacute stroke. The influence of centrally-acting medications is another factor to consider. Finally, as an emerging and potentially powerful personal characteristic, genetic polymorphisms must also be explored, as studies suggest that brain-derived neurotrophic factor val66met and apolipoprotein E $\varepsilon 4$ polymorphisms influence plasticity, stroke recovery, and responsiveness to rTMS (Cheeran et al. 2008;
Cramer et al. 2012; Kim et al. 2012b; Pattwell et al. 2012).

Regarding safety, our results showed no major adverse events. As recommended by Machii et al. (2006), we listed all symptoms reported by patients, regardless of whether the origin was thought to be related to rTMS or not. The results here with five treatments are consistent with our earlier report with one treatment (Carey et al. 2008), indicating that 6-Hz primed low-frequency rTMS applied to contralesional M1 appears to be safe.

This study has limitations. Firstly, there was no control group, which raises the concern that improvement on the TEMPA could have resulted not from the rTMS but from motor learning associated with test-retest effects and that possibly those with greater preservation of the PLIC may have been predisposed to such learning. However, our finding that significant differences in improvement persisted between responders and nonresponders even when the TEMPA paretic scores were normalized to the TEMPA nonparetic $_{\text {scores }}$ argues against motor learning. Furthermore, another study using the TEMPA in chronic stroke to measure changes in function from task-oriented motor retraining found no improvements in control subjects from baseline to posttest (Higgins et al. 2006). Secondly, although "priming" holds theoretical value for capitalizing on homeostatic plasticity mechanisms in stroke rehabilitation (Cassidy et al. In Press), at this stage we have not yet confirmed that the enhanced excitability changes found in healthy subjects with high-frequency priming prior to low-frequency rTMS (Iyer et al. 2003) also occurs in stroke. This will be an important future study for rTMS. Thirdly, the observation that training following rTMS did not influence responsiveness (Table 2) may be invalid. Only three responders and three nonresponders received rTMS+tracking training. Also, this training did not occur until the day following each rTMS session, which may have been too delayed even with priming to capitalize on any cortical excitability changes resultant from rTMS. The reason for this delay was because subjects in our initial safety study with primed rTMS (Carey et al. 2008) demonstrated fatigue immediately after the rTMS that we believed could be nonconducive to motor learning in the present study. Future rTMS studies must explore the optimal timing between rTMS and training, as a 24-hour delay may be too long but immediate training may also not be optimal because of fatigue and because of time-dependent homeostatic-like plas- 
ticity mechanisms (Fricke et al. 2011). Finally, as we did not correct for multiple statistical comparisons, our results should be interpreted with caution. We conclude that baseline paretic hand function, amount of preservation of ipsilesional PLIC, and depression score on the BDI were influential in distinguishing responders from nonresponders. Consistent with other literature, preservation of the PLIC appears to be the most influential factor affecting responsiveness to rTMS, as any upregulated excitability in ipsilesional cortical neurons stemming from rTMS-induced reduction of IHI will be to no avail if the descending projections are absent. Research into the characteristics of responders vs. nonresponders, in accompaniment with efficacy studies, must continue ambitiously to establish important decision-making guidelines that lead to improved rTMS protocols and ethical treatment for people with stroke.

\section{Acknowledgments}

This project was supported by the National Institute for Child Health and Human Development of the NIH (1 R01 HD 053153-01A2) and the National Center for Research Resources to the University of Minnesota Clinical and Translational Science Institute (CTSI) (1UL1RR033183). William Durfee, PhD, Meghan Klein, DPT; Tamara Korb, DPT; and Jamie Marsh, DPT are gratefully acknowledged for their valuable contributions to this study.

\section{References}

Ackerley, S.J., Stinear, C.M., Barber, P.A., Byblow, W.D. (2010). Combining theta burst stimulation with training after subcortical stroke. Stroke, 41, 1568-1572.

Bates, B., Choi, J.Y., Duncan, P.W., Glasberg, J.J., Graham, G.D., Katz, R.C., Lamberty, K., Reker, D., Zorowitz, R., Defense USDo, Department of Veterans, A. (2005). Veterans Affairs/Department of Defense Clinical Practice Guideline for the Management of Adult Stroke Rehabilitation Care: executive summary. Stroke, 36, 2049-2056.

Beck, A., Mendelson, M., \& Mock, J. (1961). Inventory for measuring depression. Arch Gen Psychiatry, 4, 561-571.

Berg, A., Palomaki, H., Lehtihalmes, M., Lonnqvist, J., Kaste, M. (2003). Poststroke depression: an 18-month follow-up. Stroke, 34, 138-143.

Bestmann, S., Swayne, O., Blankenburg, F., Ruff, C.C., Teo, J., Weiskopf, N., Driver, J., Rothwell, J.C., Ward, N.S. (2010). The role of contralesional dorsal premotor cortex after stroke as studied with concurrent TMS-fMRI. Journal of Neuroscience, 30, 11926-11937.

Bradnam, L.V., Stinear, C.M., Barber, P.A., \& Byblow, W.D. (2012). Contralesional hemisphere control of the proximal paretic upper limb following stroke. Cerebral Cortex, 22, 2662-2671.

Brandt, J., Benedict, R.H.B. (2001). Hopkins verbal learning test - revised. Professional Manual. Psychological Assessment Resources, Inc., Lutz, FL

Butefisch, C.M., Khurana, V., Kopylev, L., \& Cohen, L.G. (2004). Enhancing encoding of a motor memory in the primary motor cortex by cortical stimulation. Journal of Neurophysiology, 91, 2110-2116.

Carey, J.R., Evans, C.D., Anderson, D.C., Bhatt, E., Nagpal, A., Kimberley, T.J., \& Pascual-Leone, A. (2008). Safety of 6-Hz Primed Low-Frequency rTMS in Stroke. Neurorehabilitation \& Neural Repair, 22, 185-192.

Carey, J.R., Kimberley, T.J., Lewis, S.M., Auerbach, E., Dorsey, L., Rundquist, P., \& Ugurbil, K. (2002). Analysis of fMRI and Finger Tracking Training in Subjects with Chronic Stroke. Brain, 125, 773-788.

Cassidy, J.M., Gillick, B.T., Carey, J.R. (2014). Priming the Brain to Capitalize on Metaplasticity in Stroke Rehabilitation. Physical Therapy, 94, 139-150.

Cheeran, B., Talelli, P., Mori, F., Koch, G., Suppa, A., Edwards, M., Houlden, H., Bhatia, K., Greenwood, R., \& Rothwell, J.C. (2008). A common polymorphism in the brain-derived neurotrophic factor gene (BDNF) modulates human cortical plasticity and the response to rTMS.[see comment]. Journal of Physiology, 586, 5717-5725.

Chemerinski, E., Robinson, R., \& Kosier, J. (2001). Improved recovery in activities of daily living associated with remission of poststroke depression. Stroke, 32, 113-117.

Cramer, S.C., Procaccio, V., Americas, G., \& Investigators, G.I.S. (2012). Correlation between genetic polymorphisms and stroke recovery: analysis of the GAIN Americas and GAIN International Studies. European Journal of Neurology, 19, 718-724.

Dafotakis, M., Grefkes, C., Eickhoff, S.B., Karbe, H., Fink, G.R., $\&$ Nowak, D.A. (2008). Effects of rTMS on grip force control following subcortical stroke. Experimental Neurology, 211, 407-412.

Dancause, N. (2006). Vicarious function of remote cortex following stroke: recent evidence from human and animal studies. Neuroscientist, 12, 489-499.

Dassonville, P., Lewis, S.M., Zhu, X.H., Ugurbil, K., Kim, S.G., \& Ashe, J. (2001). The effect of stimulus-response compatibility on cortical motor activation. Neuroimage, 13, 1-14.

Desrosiers, J., Hebert, R., Bravo, G., Dutil, E. (1995). Upper extremity performance test for the elderly (TEMPA): normative data and correlates with sensorimotor parameters. Test d'Evaluation des Membres Superieurs de Personnes Agees. Archives of Physical Medicine \& Rehabilitation , 76, 1125-1129.

Dimyan, M.A., \& Cohen, L.G. (2010). Contribution of transcranial magnetic stimulation to the understanding of functional recovery mechanisms after stroke. Neurorehabilitation \& Neural Repair, 24, 125-135. 
Emara, T.H., Moustafa, R.R., Elnahas, N.M., Elganzoury, A.M., Abdo, T.A., Mohamed, S.A., \& Eletribi, M.A. (2010). Repetitive transcranial magnetic stimulation at $1 \mathrm{~Hz}$ and $5 \mathrm{~Hz}$ produces sustained improvement in motor function and disability after ischaemic stroke. European Journal of Neurology, 17, 12031209.

Feeney, D.M., \& Baron, J.C. (1986). Diaschisis. Stroke, 17, 817-830.

Ferbert, A., Priori, A., Rothwell, J.C., Day, B.L., Colebatch, J.G., \& Marsden, C.D. (1992). Interhemispheric inhibition of the human motor cortex. Journal of Physiology, 453, 525546.

Folstein, M., Folstein, S., \& McHugh, P. (1975). "Mini-mental state:" A practical method for grading the cognitive state of patients for the clinician. J Psychiat Res, 12, 189-198.

Fregni, F., Boggio, P.S., Valle, A.C., Rocha, R.R., Duarte, J., Ferreira, M.J., Wagner, T., Fecteau, S., Rigonatti, S.P., Riberto, M., Freedman, S.D., Pascual-Leone, A. (2006). A sham-controlled trial of a 5-day course of repetitive transcranial magnetic stimulation of the unaffected hemisphere in stroke patients. Stroke, $37,2115-2122$.

Fricke, K., Seeber, A.A., Thirugnanasambandam, N., Paulus, W., Nitsche, M.A., \& Rothwell, J.C. (2011). Time course of the induction of homeostatic plasticity generated by repeated transcranial direct current stimulation of the human motor cortex. Journal of Neurophysiology, 105, 1141-1149.

Fugl-Meyer, A., Jaasko, L., Leyman, I., Olsson, S., \& Steglind, S. (1975). The post-stroke hemiplegic patient: A method for evalulation of physical performance. Scand J Rehab Med, 7, 13-31.

Gainotti, G., Antonucci, G., Marra, C., \& Paolucci, S. (2001). Relation between depression after stroke, antidepressant therapy, and functional recovery. J Neurol Neurosurg Psychiatry, 71, 258-261.

Grefkes, C., Nowak, D.A., Wang, L.E., Dafotakis, M., Eickhoff, S.B., \& Fink, G.R. (2010). Modulating cortical connectivity in stroke patients by rTMS assessed with fMRI and dynamic causal modeling. NeuroImage, 50, 233-242.

Hackett, M.L., \& Anderson, C.S. (2005). Predictors of depression after stroke: a systematic review of observational studies. Stroke, 36, 2296-2301.

Hamzei, F., Dettmers, C., Rijntjes, M., \& Weiller, C. (2008). The effect of cortico-spinal tract damage on primary sensorimotor cortex activation after rehabilitation therapy. Experimental Brain Research, 190, 329-336.

Higgins, J., Salbach, N.M., Wood-Dauphinee, S., Richards, C.L., Cote, R., Mayo, N.E. (2006). The effect of a task-oriented intervention on arm function in people with stroke: a randomized controlled trial. Clinical Rehabilitation, 20, 296-310.

Hinder, M.R., Schmidt, M.W., Garry, M.I., \& Summers, J.J. (2010). Unilateral contractions modulate interhemispheric inhibition most strongly and most adaptively in the homologous muscle of the contralateral limb. Experimental Brain Research, 205, 423-433.

Hsu, W.-Y., Cheng, C.-H., Liao, K.-K., Lee, I.H., \& Lin, Y.-Y. (2012). Effects of repetitive transcranial magnetic stimulation on motor functions in patients with stroke: a meta-analysis. Stroke, 43, 1849-1857.
Ilic, N.V., Milanovic, S., Krstic, J., Bajec, D.D., Grajic, M., Ilic, T.V. (2011). Homeostatic modulation of stimulation-dependent plasticity in human motor cortex. Physiological Research, 60 Suppl 1, S107-112.

Iyer, M.B., Schleper, N., \& Wassermann, E.M. (2003). Priming stimulation enhances the depressant effect of low-frequency repetitive transcranial magnetic stimulation. Journal of Neuroscience, 23, 10867-10872.

Jaillard, A., Martin, C.D., Garambois, K., Lebas, J.F., \& Hommel, M. (2005). Vicarious function within the human primary motor cortex? A longitudinal fMRI stroke study. Brain, 128, 11221138.

Khedr, E.M., Abdel-Fadeil, M.R., Farghali, A., \& Qaid, M. (2009). Role of 1 and $3 \mathrm{~Hz}$ repetitive transcranial magnetic stimulation on motor function recovery after acute ischaemic stroke. European Journal of Neurology, 16, 1323-1330.

Khedr, E.M., \& Fetoh, N.A.-E. (2010). Short- and long-term effect of rTMS on motor function recovery after ischemic stroke. Restorative Neurology \& Neuroscience, 28, 545-559.

Kim, J.-M., Stewart, R., Bae, K.-Y., Kim, S.-W., Kang, H.-J., Shin, I.-S., Kim, J.-T., Park, M.-S., Kim, M.-K., Park, S.-W., Kim, Y.H., Kim, J.-K., Cho, K.-H., \& Yoon, J.-S. (2012a). Serotonergic and BDNF genes and risk of depression after stroke. Journal of Affective Disorders, 136, 833-840.

Kim, J.-M., Stewart, R., Park, M.-S., Kang, H.-J., Kim, S.-W., Shin, I.-S., Kim, H.-R., Shin, M.-G., Cho, K.-H., Yoon, J.-S. (2012b). Associations of BDNF genotype and promoter methylation with acute and long-term stroke outcomes in an East Asian cohort. PLoS ONE [Electronic Resource], 7, e51280

Kirton, A., Deveber, G., Gunraj, C., \& Chen, R. (2010). Cortical excitability and interhemispheric inhibition after subcortical pediatric stroke: plastic organization and effects of rTMS. Clinical Neurophysiology, 121, 1922-1929.

Liepert, J., Zittel, S., Weiller, C. (2007). Improvement of dexterity by single session low-frequency repetitive transcranial magnetic stimulation over the contralesional motor cortex in acute stroke: a double-blind placebo-controlled crossover trial. Restorative Neurology \& Neuroscience, 25, 461-465.

Lindenberg, R., Renga, V., Zhu, L.L., Betzler, F., Alsop, D., \& Schlaug, G. (2010). Structural integrity of corticospinal motor fibers predicts motor impairment in chronic stroke. Neurology, 74, 280-287.

Lotze, M., Markert, J., Sauseng, P., Hoppe, J., Plewnia, C., Gerloff, C. (2006). The role of multiple contralesional motor areas for complex hand movements after internal capsular lesion. Journal of Neuroscience, 26, 6096-6102.

Machii, K., Cohen, D., Ramos-Estebanez, C., \& Pascual-Leone, A. (2006). Safety of rTMS to non-motor cortical areas in healthy participants and patients. Clinical Neurophysiology, 117, 455471.

Maeda, F., Keenan, J., Tormos, J., Topka, H., Pascual-Leone, A. (2000b). Interindividual variability of the modulatory effects of repetitive transcranial magnetic stimulation on cortical excitability. Exp Brain Res, 133, 425-430.

Mansur, C.G., Fregni, F., Boggio, P.S., Riberto, M., Gallucci-Neto, J., Santos, C.M., Wagner, T., Rigonatti, S.P., Marcolin, M.A., Pascual-Leone, A. (2005). A sham stimulation-controlled trial 
of rTMS of the unaffected hemisphere in stroke patients. Neurology, 64, 1802-1804.

Moseley, A.M., \& Yap, M.C. (2003). Interrater reliability of the TEMPA for the measurement of upper limb function in adults with traumatic brain injury. Journal of Head Trauma Rehabilitation, 18, 526-531.

Murase, N., Duque, J., Mazzocchio, R., Cohen, L.G. (2004). Influence of interhemispheric interactions on motor function in chronic stroke. Annals of Neurology, 55, 400-409.

Nakagawa, S. (2004). A farewell to Bonferroni: the problems of low statistical power and publication bias. Behavioral Ecology, 15, 1044-1045.

Narushima, K., Robinson, R.G. (2003). The effect of early versus late antidepressant treatment on physical impairment associated with poststroke depression: is there a time-related therapeutic window? Journal of Nervous \& Mental Disease, 191, 645-652.

Nouri, S., \& Cramer, S.C. (2011). Anatomy and physiology predict response to motor cortex stimulation after stroke. Neurology, 77, 1076-1083

Nowak, D.A., Grefkes, C., Dafotakis, M., Eickhoff, S., Kust, J., Karbe, H., Fink, G.R. (2008). Effects of low-frequency repetitive transcranial magnetic stimulation of the contralesional primary motor cortex on movement kinematics and neural activity in subcortical stroke. Archives of Neurology, 65, 741-747.

Oldfield, R. (1971). The assessment and analysis of handedness: the Edinburgh inventory. Neuropsychologia, 9(1), 97-113.

Paolucci, S., Antonucci, G., Grasso, M., Morelli, D., Troisi, E., Coiro, P., De Angelis, D., Rizzi, F., \& Bragoni, M. (2001). Post-stroke depression, antidepressant treatment and rehabilitation results: a case-control study. Cerebrovascular Diseases, 12, 264-271.

Parikh, R.M., Robinson, R.G., Lipsey, J.R., Starkstein, S.E., Fedoroff, J.P., \& Price, T.R. (1990). The impact of poststroke depression on recovery in activities of daily living over a 2-year follow-up. Archives of Neurology, 47, 785-789.

Pattwell, S.S., Bath, K.G., Perez-Castro, R., Lee, F.S., Chao, M.V., \& Ninan, I. (2012). The BDNF Val66Met polymorphism impairs synaptic transmission and plasticity in the infralimbic medial prefrontal cortex. Journal of Neuroscience, 32, 2410-2421.

Pocock, S.J. (1997). Clinical trials with multiple outcomes: a statistical perspective on their design, analysis, and interpretation. Controlled Clinical Trials, 18, 530-545; discussion 546-539.

Pohjasvaara, T., Vataja, R., Leppavuori, A., Kaste, M., \& Erkinjuntti, T. (2001). Depression is an independent predictor of poor long-term functional outcome post-stroke. European Journal of Neurology, 8, 315-319.

Riley, J.D., Le, V., Der-Yeghiaian, L., See, J., Newton, J.M., Ward, N.S., Cramer, S.C. (2011). Anatomy of stroke injury predicts gains from therapy. Stroke, 42, 421-426.

Robinson, R.G., \& Spalletta, G. (2010). Poststroke depression: a review. Canadian Journal of Psychiatry - Revue Canadienne de Psychiatrie, 55, 341-349.

Rossini, P.M., \& Pauri, F. (2000). Neuromagnetic integrated methods tracking human brain mechanisms of sensorimotor areas 'plastic' reorganisation. Brain Research - Brain Research Reviews, $33,131-154$
Schaechter, J.D., Perdue, K.L., \& Wang, R. (2008). Structural damage to the corticospinal tract correlates with bilateral sensorimotor cortex reorganization in stroke patients. NeuroImage, $39,1370-1382$.

Schulz, R., Park, C-H, Boudrias, M-H, Gerloff, C., Hummel, F.C., \& Ward, N.S. (2012). Assessing the integrity of corticospinal pathways from primary and secondary cortical motor areas after stroke. Stroke, 43, 2248-2251.

Seniów, J.B.M., Lesniak, M., Waldowski, K., Iwanski, S., \& Czlonkowska, A. (2012). Transcranial magnetic stimulation combined with physiotherapy in rehabilitation of poststroke hemiparesis: a randomized, double-blind, placebo-controlled study. Neurorehabil Neural Repair, 26, 1072-1079.

Siebner, H.R., Lang, N., Rizzo, V., Nitsche, M.A., Paulus, W., Lemon, R.N., \& Rothwell, J.C. (2004). Preconditioning of LowFrequency Repetitive Transcranial Magnetic Stimulation with Transcranial Direct Current Stimulation: Evidence for Homeostatic Plasticity in the Human Motor Cortex. J. Neurosci, 24, 3379-3385.

Stinear, C.M., Barber, P.A., Petoe, M., Anwar, S., \& Byblow, W.D. (2012). The PREP algorithm predicts potential for upper limb recovery after stroke. Brain, 135, 2527-2535.

Stinear, C.M., Barber, P.A., Smale, P.R., Coxon, J.P., Fleming, M.K., \& Byblow, W.D. (2007). Functional potential in chronic stroke patients depends on corticospinal tract integrity. Brain, 130, 170-180.

Takeuchi, N., Chuma, T., Matsuo, Y., Watanabe, I., \& Ikoma, K. (2005). Repetitive Transcranial Magnetic Stimulation of Contralesional Primary Motor Cortex Improves Hand Function After Stroke. Stroke, 36, 2681-2686.

Takeuchi, N., Tada, T., Toshima, M., Chuma, T., Matsuo, Y., \& Ikoma, K. (2008). Inhibition of the unaffected motor cortex by $1 \mathrm{~Hz}$ repetitive transcranical magnetic stimulation enhances motor performance and training effect of the paretic hand in patients with chronic stroke. Journal of Rehabilitation Medicine, 40, 298-303.

Talelli, P., Greenwood, R.J., \& Rothwell, J.C. (2006). Arm function after stroke: neurophysiological correlates and recovery mechanisms assessed by transcranial magnetic stimulation. Clinical Neurophysiology, 117, 1641-1659.

Talelli, P., Wallace, A., Dileone, M., Hoad, D., Cheeran, B., Oliver, R., VandenBos, M., Hammerbeck, U., Barratt, K., Gillini, C., Musumeci, G., Boudrias, M.-H., Cloud, G.C., Ball, J., Marsden, J.F., Ward, N.S., Di Lazzaro, V., Greenwood, R.G., Rothwell, J.C. (2012). Theta burst stimulation in the rehabilitation of the upper limb: a semirandomized, placebo-controlled trial in chronic stroke patients. Neurorehabilitation \& Neural Repair, 26, 976-987.

Theilig, S., Podubecka, J., Bosl, K., Wiederer, R., Nowak, D.A. (2011). Functional neuromuscular stimulation to improve severe hand dysfunction after stroke: does inhibitory rTMS enhance therapeutic efficiency? Experimental Neurology, 230, $149-155$.

Trotti, A., Colevas, A.D., Setser, A., Rusch, V., Jaques, D., Budach, V., Langer, C., Murphy, B., Cumberlin, R., Coleman, C.N., \& Rubin, P. (2003). CTCAE v3.0: development of a comprehensive grading system for the adverse effects of cancer treatment. Seminars in Radiation Oncology, 13, 176-181. 
Ward, N.S., Newton, J.M., Swayne, O.B., Lee, L., Thompson, A.J., Greenwood, R.J., Rothwell, J.C., \& Frackowiak, R.S. (2006). Motor system activation after subcortical stroke depends on corticospinal system integrity. Brain, 129, 809-819.

Yousry, T.A., Schmid, U.D., Alkadhi, H., Schmidt, D., Peraud, A., Buettner, A., \& Winkler, P. (1997). Localization of the motor hand area to a knob on the precentral gyrus. A new landmark. Brain, 120, 141-157.

Zhu, L.L., Lindenberg, R., Alexander, M.P., \& Schlaug, G. (2010). Lesion load of the corticospinal tract predicts motor impairment in chronic stroke. Stroke, 41, 910-915. 